INPLASY

PROTOCOL

To cite: Du et al. Systematic review and Meta-analysis of transdermal preparations of Sinomenium acutum in rheumatoid arthritis. Inplasy protocol 202160085. doi: 10.37766/inplasy2021.6.0085

Received: 23 June 2021

Published: 23 June 2021

Corresponding author: Sisi Du

dss1005@163.com

Author Affiliation: LongHua Hospital,Shanghai University of Traditional Chinese Medicine.

Support: Shanghai Municipal Health Bure.

Review Stage at time of this submission: Formal screening of search results against eligibility criteria.

Conflicts of interest: None declared.

\section{Systematic review and Meta-analysis of transdermal preparations of Sinomenium acutum in rheumatoid arthritis}

Du, SS 1; Fu, QH2; Pei, J3; Zhou, H4.

Review question / Objective: To systematically evaluate the curative clinical efficacy and safety of transdermal preparations of Sinomenium acutum (SA) in the treatment of rheumatoid arthritis (RA).

Condition being studied: Rheumatoid arthritis (RA) is an autoimmune disease with symmetrical polyarthritis as the main clinical manifestation, characterized by chronic synovial inflammation and progressive destruction of joints. At present, the number of patients accounts for about $1.0 \%$ of the world's total population, and the prevalence rate in China is $0.32 \%-0.34 \%$, which is one of the main causes of the loss of labor force and disability. It can occur at any age, the high incidence age for women is 45 54 years old, and the incidence rate for men increases with age. The ratio of men and women suffering from RA is about 1: 3.To systematically evaluate the curative clinical efficacy and safety of transdermal preparations of Sinomenium acutum (SA) in the treatment of RA.

INPLASY registration number: This protocol was registered with the International Platform of Registered Systematic Review and Meta-Analysis Protocols (INPLASY) on 23 June 2021 and was last updated on 23 June 2021 (registration number INPLASY202160085).

\section{INTRODUCTION}

Review question / Objective: To systematically evaluate the curative clinical efficacy and safety of transdermal preparations of Sinomenium acutum (SA) in the treatment of rheumatoid arthritis (RA).

Condition being studied: Rheumatoid arthritis (RA) is an autoimmune disease with symmetrical polyarthritis as the main 
clinical manifestation, characterized by chronic synovial inflammation and progressive destruction of joints. At present, the number of patients accounts for about $1.0 \%$ of the world's total population, and the prevalence rate in China is $0.32 \%-0.34 \%$, which is one of the main causes of the loss of labor force and disability. It can occur at any age, the high incidence age for women is 45 54 years old, and the incidence rate for men increases with age. The ratio of men and women suffering from RA is about 1: 3.To systematically evaluate the curative clinical efficacy and safety of transdermal preparations of Sinomenium acutum (SA) in the treatment of RA.

\section{METHODS}

Participant or population: The included participants were patients with a clear diagnosis of rheumatoid arthritis.

Intervention: Sinomenine injection with transdermal method or Sinomenium acutum containing external preparation combined with disease modifying antirheumatic drugs.

Comparator: Disease modifying antirheumatic drugs(DMARDs) only.

Study designs to be included: Randomized controlled trials.

Eligibility criteria: (1) The included participants were patients with a clear diagnosis of RA; (2) The subjects took SIN injection with transdermal method or SA containing external preparation combined with DMARDs versus DMARDs only;(3) Outcome indicators contains total effective rate, VAS, morning stiffness time, erythrocyte sedimentation rate (ESR), Creactive protein (CRP), rheumatoid factor (RF) ,DAS28 and Safety evaluation; (4) Randomized controlled trials (RCTs).

Information sources: CNKI, Wanfang Data, SinoMed, VIP, PubMed and Cochrane library.

Main outcome(s): Total effective rate.
Additional outcome(s): Visual Analogue Scale(VAS), morning stiffness time, erythrocyte sedimentation rate (ESR), Creactive protein (CRP), rheumatoid factor (RF) ,DAS28 and Safety evaluation.

Quality assessment / Risk of bias analysis: The quality of each study included in this review was assessed using the Cochrane Handbook for Systematic Reviews of Interventions. The details that were assessed as follows: (1) random allocation method; (2) hidden grouping; (3) blind method; (4) result data integrity; (5) selective reporting of research results; (6) other sources of bias. For each included study, the above six items were evaluated as "low" (representing low risk of bias), "high" (representing high risk of bias) or "unclear" (representing moderate or unknown risk of bias).

Strategy of data synthesis: Review Manager (Revman) (Computer program), version 5.4.1, was used to analyze the research data. The binary variables were expressed as odds ratio (OR) and $95 \% \mathrm{Cl}$. For continuous variables, mean difference (MD) or standardized mean difference (SMD) were selected as the combined statistics, and each effect quantity was expressed with $95 \% \mathrm{Cl}$. Analysis was carried out using a fixed or random effects model according to the heterogeneity. The percentage of heterogeneity in the study was determined by the 12 statistic, with a value of $0 \%$ indicating no heterogeneity and larger values indicating increased heterogeneity. A $P$ value $<0.10$ was considered to suggest statistical heterogeneity and prompted random effects modelling.

Subgroup analysis: Each of outcome will be conducted subgroup if necessary.

Sensitivity analysis: None planed.

Country(ies) involved: China.

Keywords: Sinomenium acutum;

Sinomenine; transdermal drug delivery 
system(TDDS); rheumatoid arthritis;

systematic review.

Contributions of each author:

Author 1 - SISI DU.

Email: dss1005@163.com

Author 2 - QINHUI FU.

Email: ff777315@163.com

Author 3 - JIAN PEI.

Author 4 - HUA ZHOU. 